\title{
Determining Window Placement and Configuration for the Small Pressurized Rover (SPR)
}

\section{Shelby Thompson, Ph.D.}

$$
\text { Harry "Chip" Litaker }
$$

Usability Testing and Analysis Facility (UTAF)

Lockheed Martin

$$
\text { Robert Howard, Ph.D. }
$$

Johnson Space Center, NASA 


\section{Contributors}

\section{Rich Szabo}

Habitability Design Center (HDC)

Lockheed Martin
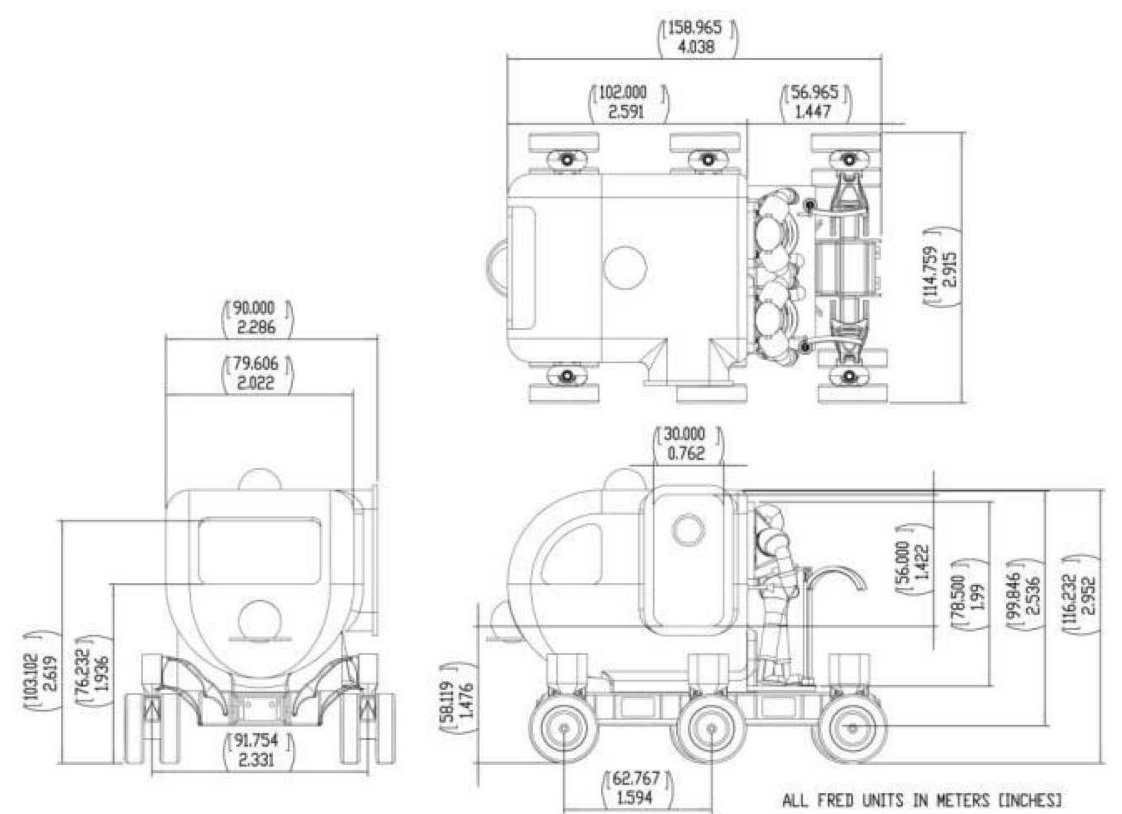

\section{Evan Twyford \\ Carl "Chip" Conlee}

Habitability Design Center (HDC)

MEI Technologies

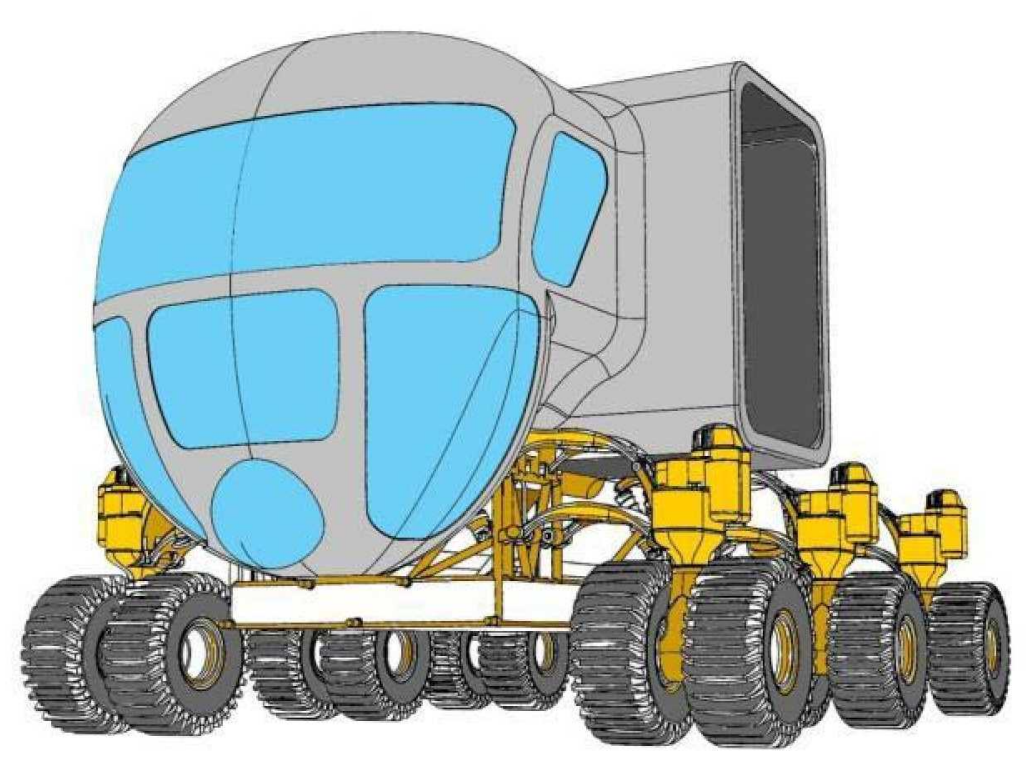




\section{Background}

- The rover began development in 2007 in order to test the concept of a multiple wheeled vehicle that had the ability to move in any direction upon the lunar surface

- Allows the user to "keep an eye" on a target while being able to navigate over various terrains

- Two versions were designed using the same chassis (a) an unpressurized "Apollo"-style rover and (b) a small pressurized rover (since become known as the Lunar Electric Rover, LER)
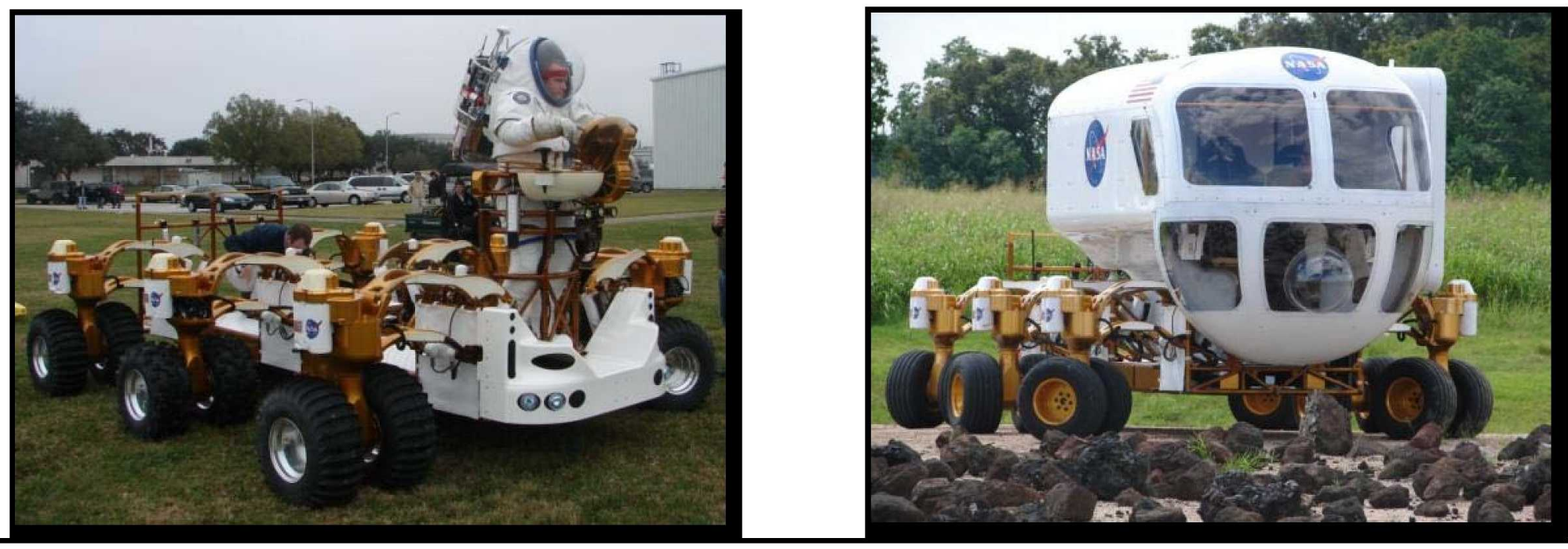


\section{Background}

- An enclosed cabin was suggested that used suit ports to enter and exit the vehicle for EVA, includes a side hatch that would be used docked to a Lunar outpost or another rover
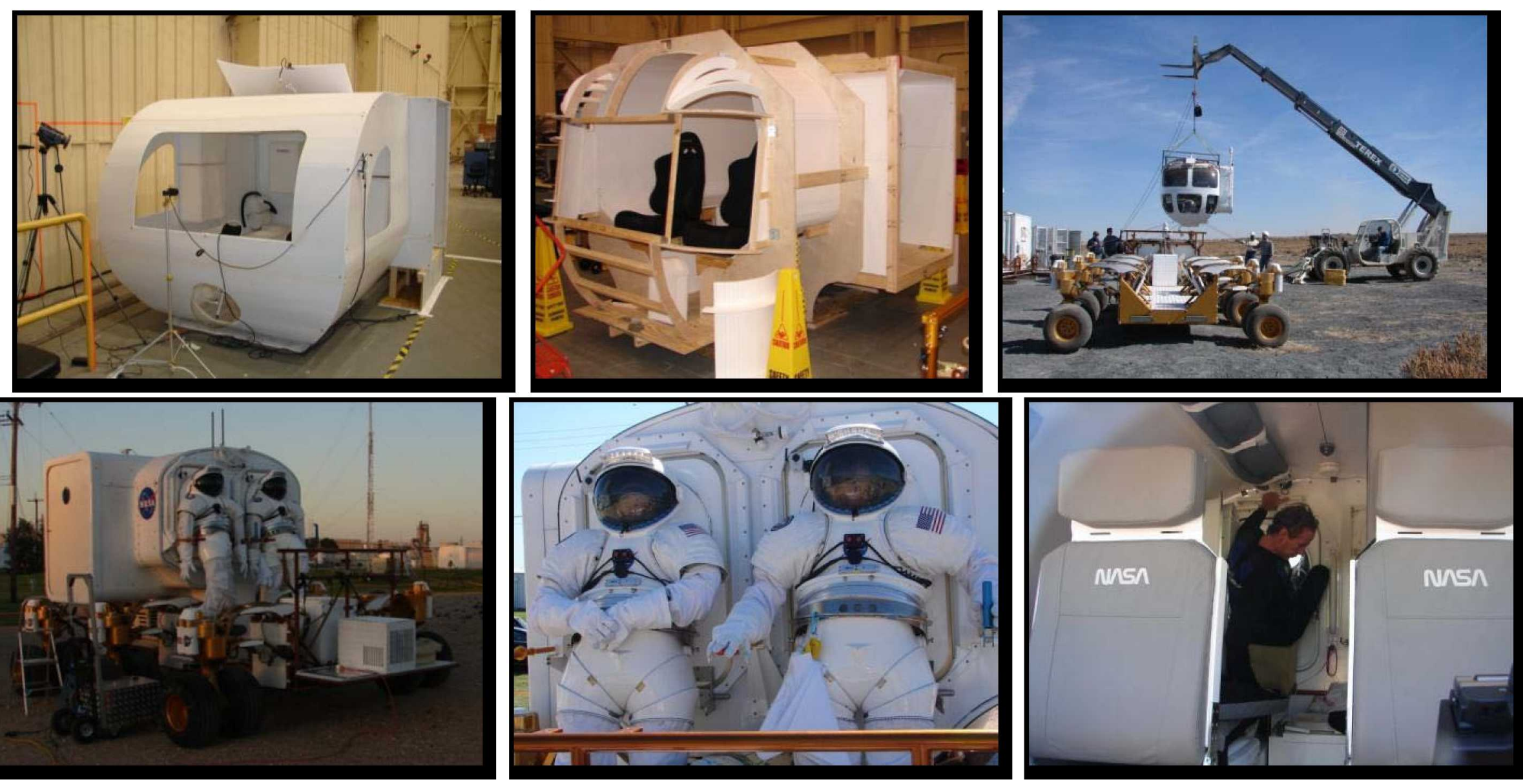

Page No. 4 


\section{Purpose of Evaluation}

- The purpose of the evaluation was to obtain human-in-the-loop data on window placement and configuration for the cockpit of the LER

- A baseline window placement and configuration was developed from the results

- This baseline does not reflect a flight-rated vehicle and ignores critical issues such as structural integrity, thermal factors, weight, mass, etc.

- A decision was made not to limit the participants in where they could place and configure the windows

- It was assumed that given the landscape of the lunar surface that persons would want a wide forward views for navigation over and around objects, sides windows for docking maneuvers, lower/corner views for obstacle avoidance at the wheels, and lower/forward views for geological observations 


\section{Method of Evaluation}

- $\quad$ Nine SMEs participated (7 males and 2 females)

- Astronauts, geologists, and engineers

- Each was experienced with current and prior LER configurations

The evaluation took place in the Reconfigurable Operational Cockpit (ROC) at Johnson Space Center, NASA

- Ability to project a 2-D image on a domed screen

- A medium to high resolution lunar environment was developed using actual lunar images of the surface

- Due to the size of the LER mockup in the dome, no side views of the images were available

- Participants sat in the left cockpit seat and controlled a joystick mounted on the right to "drive" the vehicle over the lunar surface

- The projection included flat to mountainous terrain with craters, rocks, and boulders 


\section{Method of Evaluation}

- The LER mockup was constructed of wood and formcore board

- Several options were considered to evaluated the placement and configuration of the windows (i.e., head and eye-tracker), but were rejected for several reasons

- Lack of portability of the head and eye-tracking system

- Not enough led-time to develop the cockpit's "virtual bubble" that would simulate the surface of the LER's front-nose section

- Environment was not optimal for video camera use (dim to low lighting) and structure did not support the use of a magnetic head tracker (metal used in the mockup and the ROC)

- Decision was made to create a "grid" that covered the entire front cockpit with letters and numbers to denote a specific area of the grid 

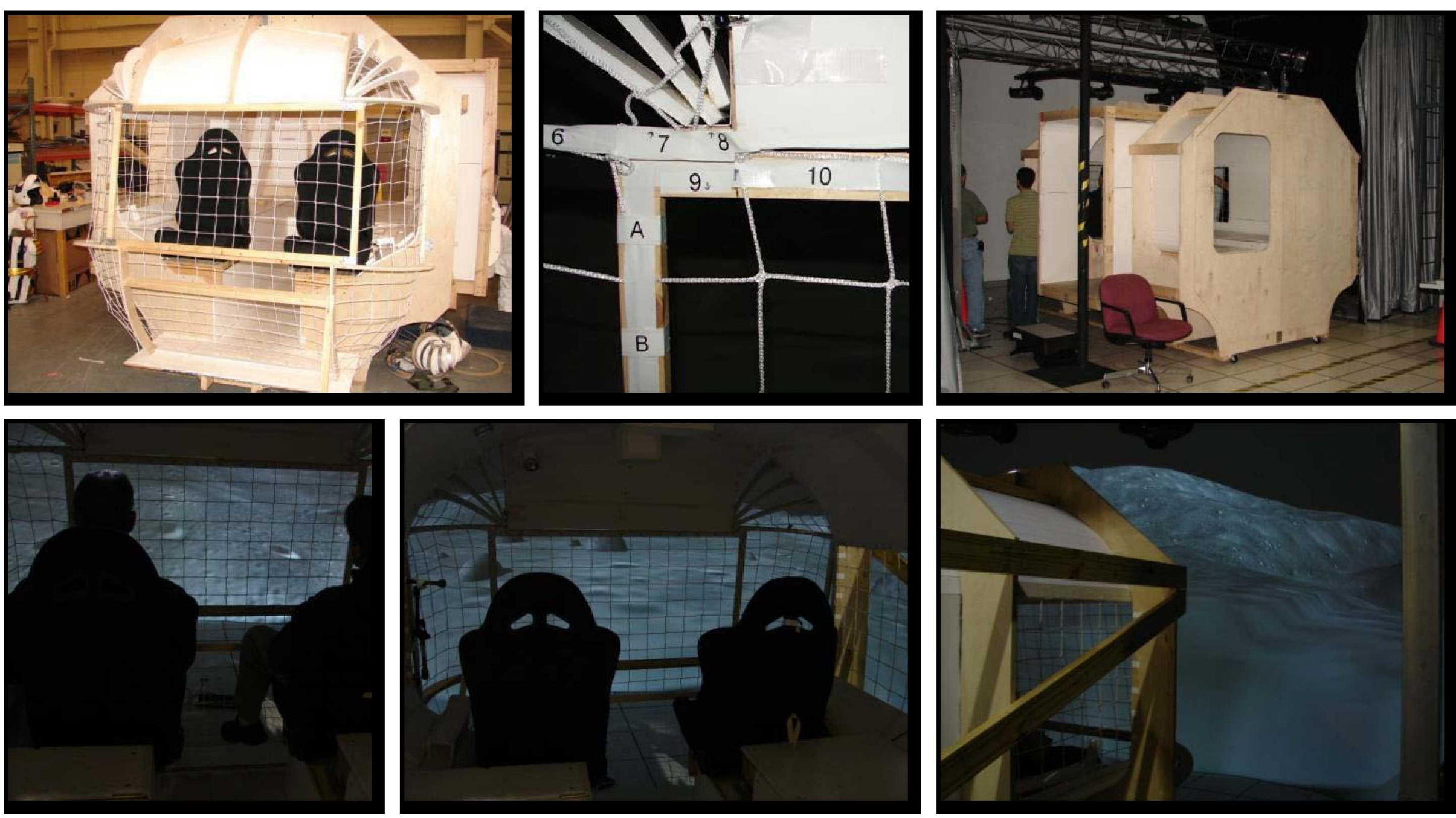


\section{Data Collection}

- Using combined images of the cockpit, from the POV as if you were sitting in the center of the vehicle, a composite image was created and printed on transparency

- Participants colored in areas they felt were necessary areas for windows - red (high priority/must have), green (like to have), and blue (low priority)

- Ten areas were rated ( $1=$ unnecessary to $5=$ necessary $)$ in terms of necessity
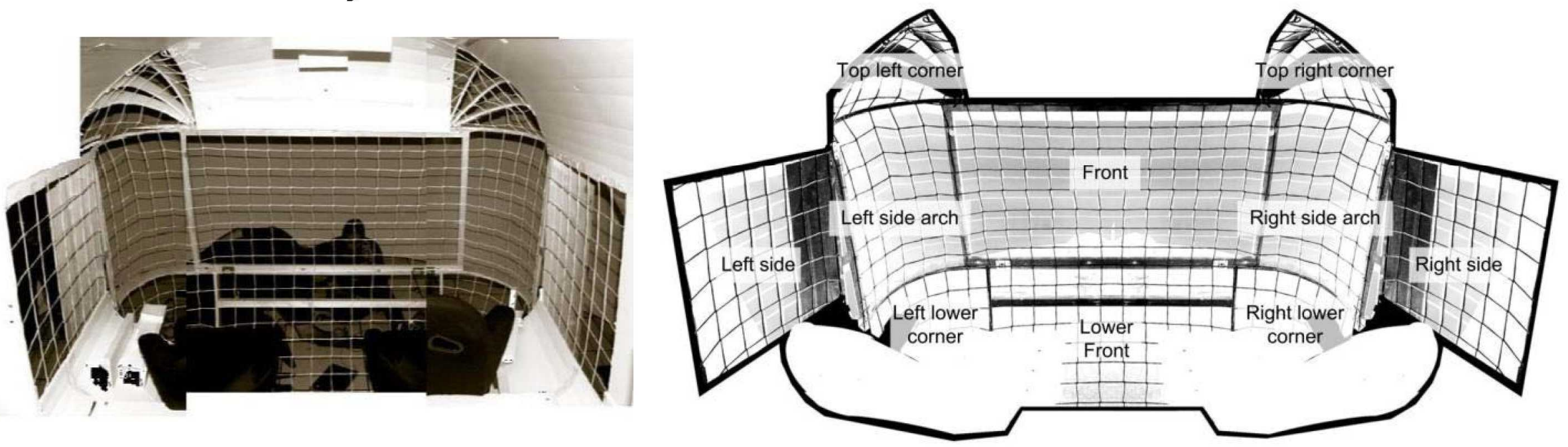


\section{Results}

- Different areas were rated as more necessary depending on the simulated terrain tested

- For example, upper corners were rated more necessary for mountainous terrain, while sides were rated high for rock avoidance and crater inclines

Means for Task by Rover Area for Necessity of Windows

\begin{tabular}{lcccccc}
\hline \multicolumn{1}{c}{ Task } & Upper Corners & Sides & Side Arch & Front & Lower Corners & Lower Front \\
\hline Smooth Flat & 1.67 & 1.50 & 4.44 & 5.00 & 1.44 & 1.33 \\
Hilly Terrain & 1.44 & 1.78 & 4.56 & 5.00 & 2.11 & 1.44 \\
Mountain Terrain & 3.50 & 1.89 & 5.00 & 5.00 & 1.67 & 1.56 \\
General Rock Field & 1.00 & 2.00 & 4.89 & 5.00 & 2.67 & 2.22 \\
Rock Avoidance & 1.00 & 2.28 & 4.94 & 5.00 & 2.67 & 1.67 \\
Circling Rock & 1.00 & 1.38 & 4.69 & 5.00 & 2.13 & 1.88 \\
Crater Incline & 2.13 & 3.00 & 4.88 & 5.00 & 2.13 & 1.88 \\
Overall Rating & 2.22 & 2.78 & 4.89 & 5.00 & 2.67 & 2.33 \\
Grand Mean & 1.75 & 2.07 & 4.79 & 5.00 & 2.19 & 1.79 \\
\hline
\end{tabular}

Note. $1=$ unnecessary, $3=$ neutral, $5=$ necessary. 


\section{Results}

- We took the intersection, or congruent, of all red, green, and blue areas to determine the approximate placement and configuration of the windows
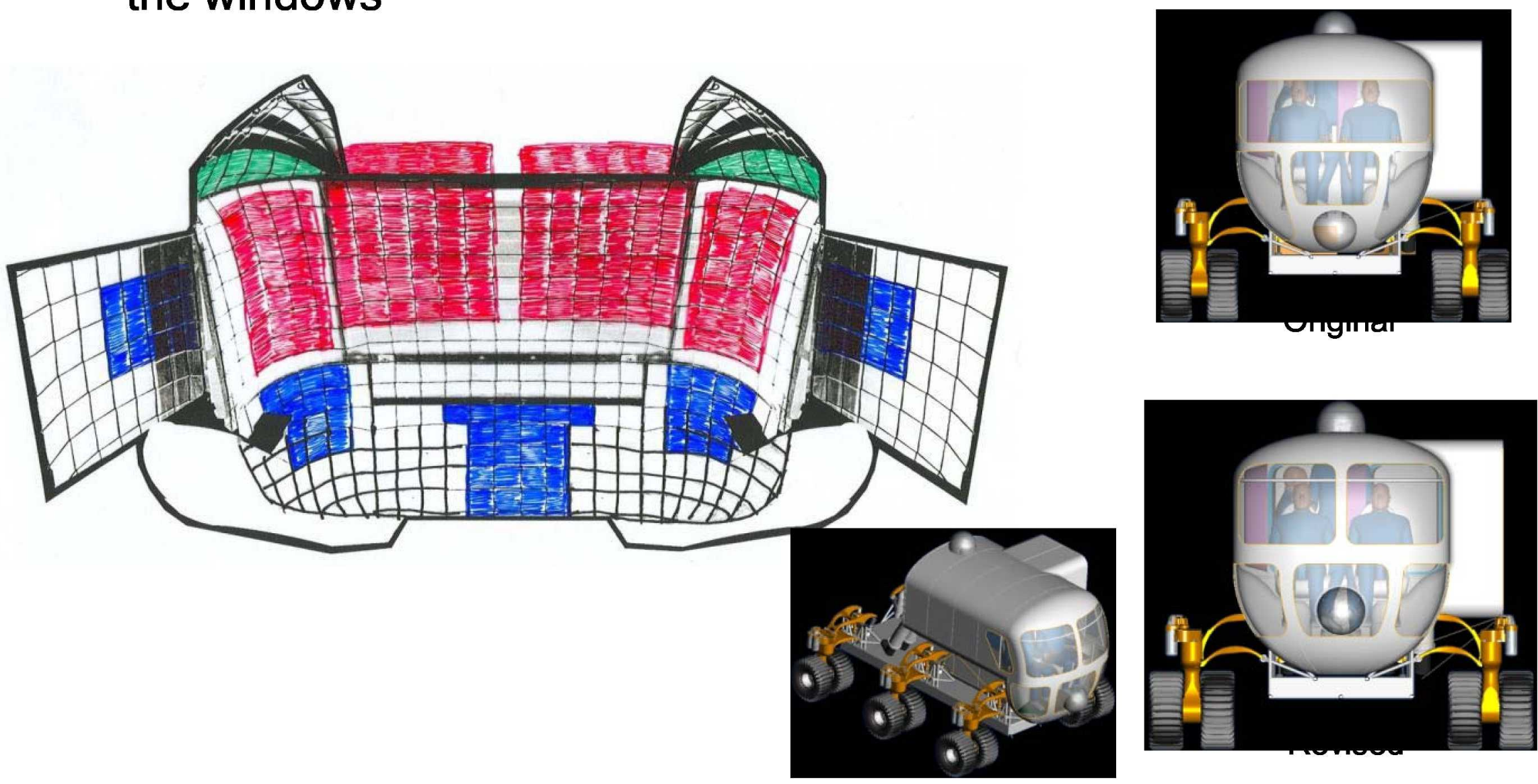


\section{Where is the LER now?}

- Since this study, the cabin 1a was developed, created, and tested

- A revised cabin $1 \mathrm{~b}$ has designed, build, and tested based on human factors recommendations
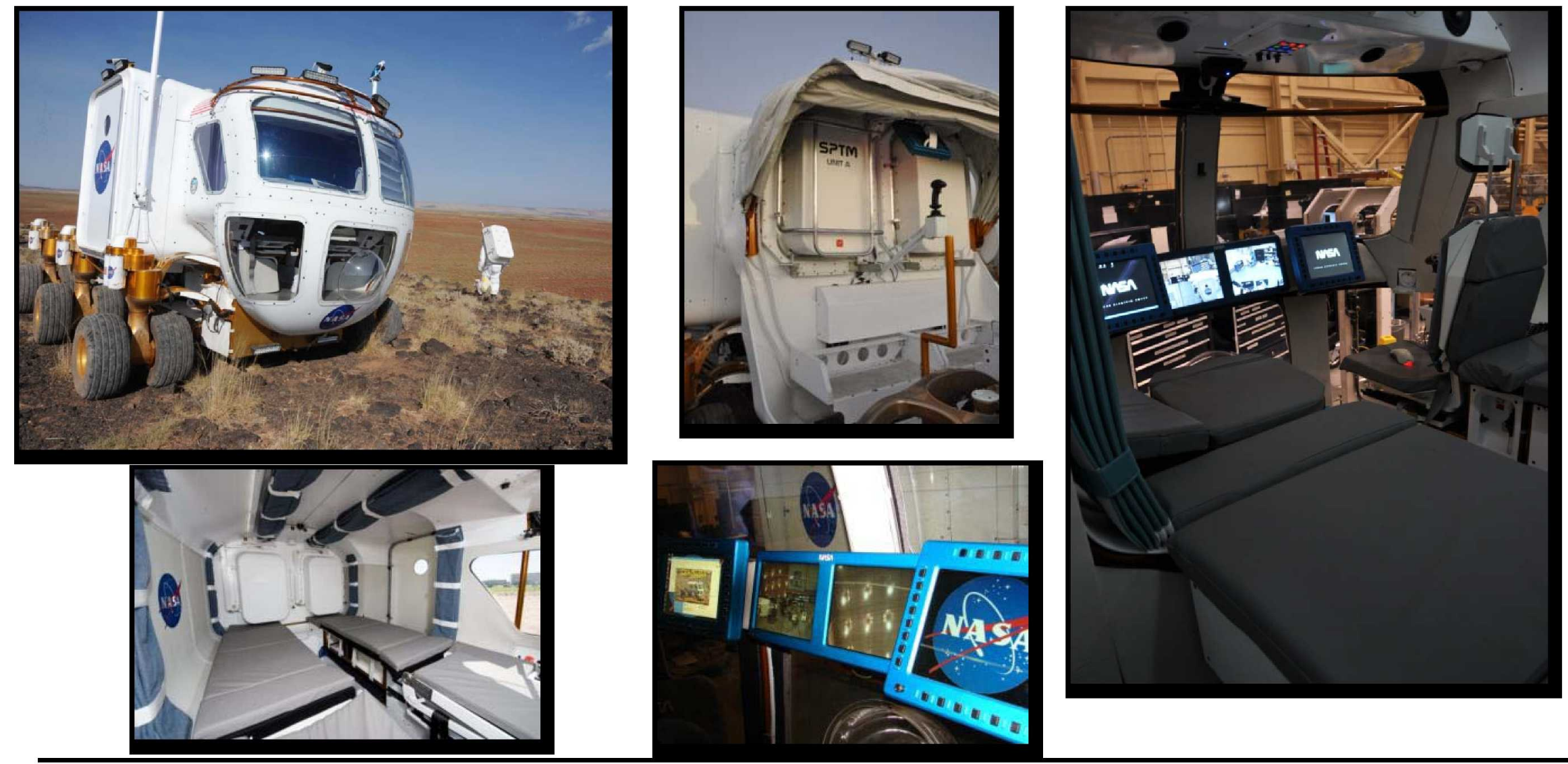


\section{Thank You}

- Questions?
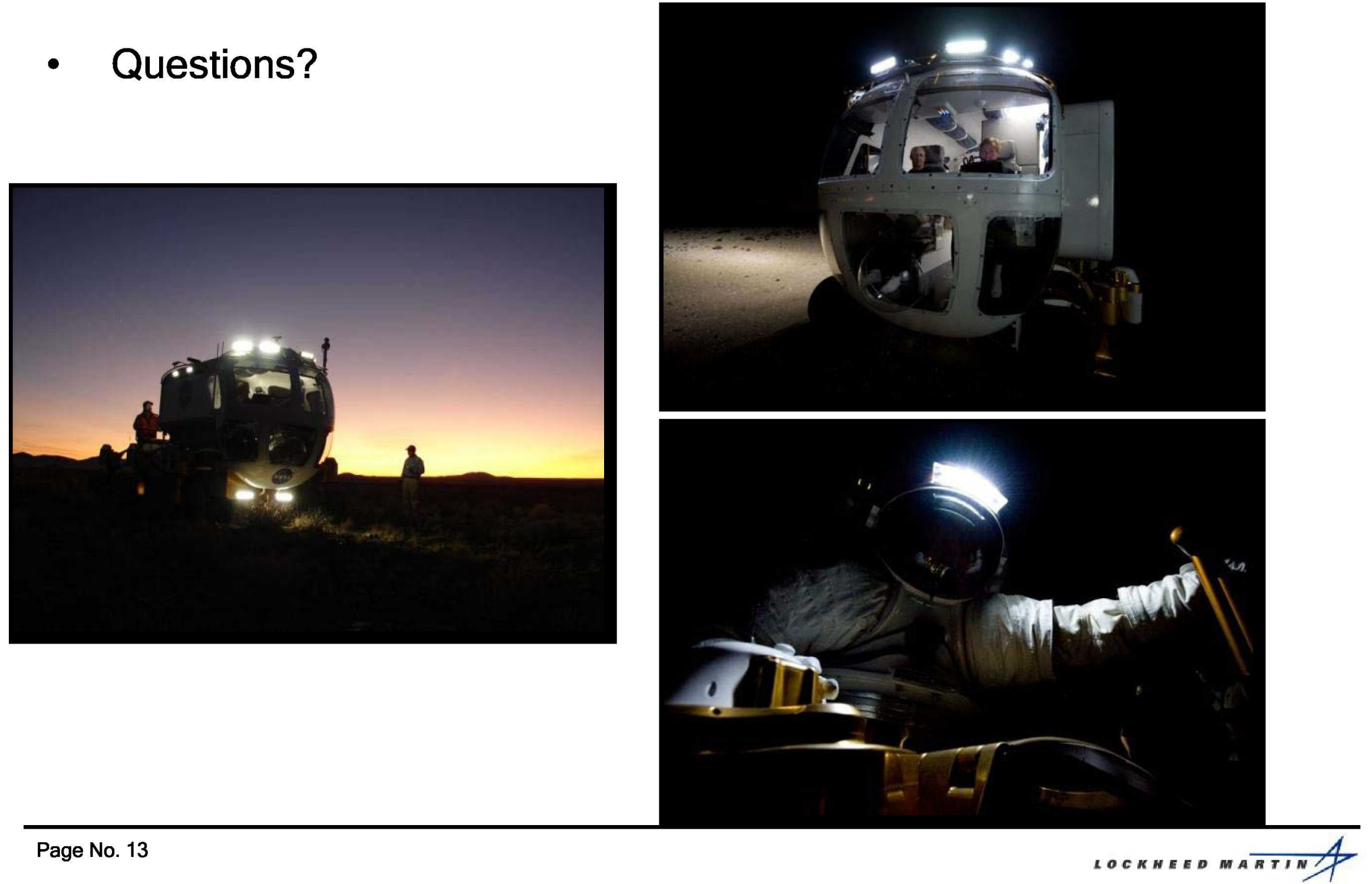